Convergences francophones $5.2(2017): 42-58$

http://mrujs.mtroyal.ca/index.php/cf/index

\title{
Monstruosités et esthétiques du chaos dans les dramaturgies contemporaines africaines
}

Sylvie Ngilla

Université de San Diego

L'histoire de la création dramatique africaine francophone depuis la fin des colonisations s'est construite dans le processus permanent d'une fabrique de l'identité. Dans son introduction générale sur l'évolution historique du théâtre noir francophone, Louise M. Jefferson identifie une intensification des débats littéraires sur l'identité africaine et l'africanité dans les années 1970 et 1980 qui tirent les leçons et bilans de la période des indépendances africaines. La multiplication des rencontres académiques et artistiques à l'instar du «Colloque sur le théâtre négroafricains » de 1970 en Côte d'Ivoire, le « Colloque sur Le Critique africain et son peuple comme producteur de civilisation » de 1973 au Cameroun ou encore celui organisé au Mali en 1988 intitulé «Théâtre africain, Théâtres africains ?» témoignent d'une réflexion continue sur les conditions de possibilité du renouvellement de la critique et de l'identité littéraire et artistique africaine. Selon Jefferson, les débats contemporains sur le théâtre noir francophone sont organisés principalement à partir de deux grandes tendances, traditionnaliste et libertaire. La tendance traditionnaliste regroupe les critiques, théoriciens et auteurs du théâtre noir francophone «qui croient à la nécessité d'ancrer toute expression théâtrale dans les traditions formelles d'antan tout en respectant l'apport créateur ou meneur de jeu » (12). La tendance libertaire au contraire est incarnée par « ceux qui sont partisans d'une liberté créatrice absolue accordée à l'auteur sans qu'il/elle se sente entravé/e par l'idéologie et les pratiques d'autrefois » (12). Cette dichotomie est loin d'être hermétique puisque les esthétiques théâtrales négro-africaines revendiquent leur liberté artistique dans la réactualisation des traditions, contes et rites africains. Werewere Liking, figure emblématique du renouveau de l'esthétique du théâtre-rituel depuis les années 1980, modernise les rituels bassa du Cameroun à partir du travail collectif de sa compagnie Ki-Yi Mbock. L'opposition entre ceux qui défendent un théâtre africain plus ancré dans les traditions et ceux qui mettent l'accent sur une liberté absolue des créateurs africains ne se situe pas dans l'enjeu compétitif d'une échelle de valeur. Elle met en évidence la radicalisation d'une nouvelle vague de dramaturges africains qui déplace le processus identitaire dans les confins d'une esthétique du chaos et de la monstruosité.

L'émergence de cette nouvelle vague de dramaturgies africaines francophones depuis les années 1990 est identifiée chez des auteurs tels que Koffi Kwahulé (Côte d'Ivoire), Kossi Efoui (Togo), José Pliya (Bénin), Dieudonné Niangouna (Congo), Marcel Zang (Cameroun) parmi d'autres qui revendiquent une liberté de création affranchie des codes conventionnels de lecture. Ces dramaturges participent et prolongent le débat sur la construction de l'identité africaine dans la création littéraire et artistique mais à partir de points de vue subversifs qui exploitent les notions d'hybridité et de transformation permanente. "Habitants 
d'un théâtre en mutation » (3) selon l'expression de Caya Makhélé dans un numéro consacré aux "Théâtres d'Afrique noire", ces écrivains produisent un théâtre traversé par des personnages monstres nourris par des référents culturels multiples et une esthétique du chaos. La définition classique du monstre et du chaos qui renvoient aux défis des normes naturelles et sociétales permet d'éclairer les discours de protestation de cette génération. Contre les accusations sur le manque d'africanité dans ses pièces de théâtre, le dramaturge Koffi Kwahulé évoque «le syndrome Frankenstein » dans une table ronde ${ }^{1}$ dans laquelle il explique la dérive néocoloniale et exotique de certains critiques littéraires qui enferment les artistes africains dans une identité stéréotypée et close. L'ouvrage de Sylvie Chalaye, Afrique noire et dramaturgies contemporaines: le syndrome Frankenstein, qui reprend de manière stratégique la formule de Kwahulé consiste à analyser les facettes du monstre intérieur revendiqué chez cette nouvelle génération. Chalaye résume ainsi le projet de son ouvrage : «Briser l'enclos, s'engager dans une traversée, assumer l'aliénation historique et la transcender en faisant cap sur le monde, telles seront les quatre étapes de ce cheminement, les quatre entrées que nous proposons pour autopsier l'alien qui habite ces dramaturgies africaines contemporaines $»(14)$.

Notre étude s'appuie sur l'analyse générale de Chalaye mais nous poursuivons l'interrogation du sens de l'hybridité en tant que processus d'identité et d'écriture dramatique dans le contexte des dramaturgies de la diaspora africaine d'expression française. L'enjeu consiste à identifier les conditions de production des identités-monstres dans le cadre d'une théorisation du chaos chez une nouvelle génération d'auteurs africains.

\section{Constructions hybrides}

Le terme "hybride" dont l'étymologie latine ibrida signifie de sang mêlé est à l'origine utilisé dans la botanique et la biologie animale pour définir le croisement de deux espèces. Les mythologies et littératures à travers le monde ont cependant toujours été peuplées de créatures et divinités hybrides composées de parties du corps humain dont la forme monstrueuse évoque des sentiments de peur, d'horreur, de beauté ou de fascination. Parmi quelques exemples citons le Minotaure, créature hybride mi-homme mi-taureau dans la mythologie grecque, Mamiwata, la Mère des eaux et divinité africaine mi-femme, mi-poisson, le Sphinx, représenté souvent par une tête humaine pharaonique sur le corps d'un lion dans les mythologies égyptiennes ou encore le Garuda, créature divine mi-homme mi-aigle dans les traditions hindouistes et bouddhistes. L'époque des empires coloniaux à partir du XVII ${ }^{\mathrm{e}}$ siècle a accentué un sens péjoratif de l'hybridité en tant que bâtardise et référence aux personnes métissées, les "mulâtres", mot dérivé de mulet ou mule, l'animal hybride issu du croisement entre l'âne et la jument. C'est à partir des études postcoloniales autour des années 1980 et 1990 que l'hybridité en tant que concept culturel et identitaire est redéfinie comme espace positif de transformation

${ }^{1}$ Table ronde « Dramaturgies africaines d'aujourd'hui. » Les petits matins du TOMA, La Chapelle du Verbe incarné, Avignon, le 23 juillet 2001. 
et d'émancipation à partir entre autre des théories de Gayatri C. Spivak et de Homi K. Bhabha. Dans son célèbre essai, The Location of Culture (1994). Bhabha propose de sortir du paradigme colonial de la représentation de l'Autre qui perpétue des stéréotypes sur l'identité et une logique d'exploitation occidentale depuis le XVII ${ }^{\mathrm{e}}$ siècle. Bhabha incarne le projet des études postcoloniales dans la volonté d'établir une nouvelle organisation du monde et réinventer un rapport à l'Autre à partir d'une pensée de l'hybridité culturelle. Cette hybridité culturelle ne s'entend pas dans la définition classique du croisement de deux espèces. Elle constitue un "tiers espace" en tant que résistance culturelle émergeante au sein de l'ambivalence de l'idéologie coloniale qui est marquée à la fois par l'injonction au mimétisme et le rejet de l'Autre.

Un contexte différent qui néanmoins partage des similitudes avec les études postcoloniales voit l'émergence de nouvelles dramaturgies contemporaines qui remettent en cause le paradigme identitaire africain hérité du pouvoir colonial mais aussi des discours essentialistes et nationalistes. Depuis les années 1990, plusieurs voix dans le paysage littéraire africain affirment l'hybridité comme champ de possibilités transculturelles dans la construction de l'identité et de la création littéraire et artistique. Contre les discours essentialistes visant à établir les degrés d'africanité d'une œuvre, une vague de dramaturges africains a farouchement opposé des déclarations et textes à valeur de manifestes qui célèbrent l'espace chaotique de l'identité monstre. À la critique de Françoise Gründ dans son article intitulé "La Parole lourde des théâtres africains en Afrique noire" qui reproche le manque d'africanité de certaines œuvres dramatiques issues notamment de la diaspora africaine qu'elle considère trop occidentalisées, Kossi Efoui rétorque avec ironie dans la préface de sa pièce L'entre-deux rêves de Pitagaba conté sur les trottoirs de la radio: «Une quête du typique qui autorise à dire que tel spectacle n'est pas africain ou que tel auteur gagnerait à être moins occidentalisé. On est tenté de répondre : à partir de combien de plumes au cul la chose est-elle crédible ? À moins que ce soit à partir d'un degré supposé de régression au stade oral » (7-8).

Koffi Kwahulé dénonce également le sectarisme d'une critique bienpensante dans son article "Quand l'Afrique dérive vers l'intégrisme culturel" dans lequel il critique le confinement des littératures africaines dans des perspectives culturelles et raciales au détriment de l'analyse de la qualité littéraire et des formes esthétiques.

Si l'on se réfère aux déclarations des novateurs, les critères d'africanité se cristallisent autour de la musique, de la danse, du recours aux formes traditionnelles authentiquement négro-africaines et du concept d'art total. Or, il se trouve que tout cela n'a rien «d'authentiquement africain ». Outre le fait que l'efficacité de la totalisation de tous les arts reste à démontrer, rappelons qu'elle n'a rien d'exclusivement négro-africain et que sur tous les continents, à ce moment ou à un autre, les hommes l'ont pratiquée. Le théâtre balinais, le kabuki, les théâtres rituels d'Océanie, le Kathakali... relèvent tous de la même volonté totalisatrice. ... Il est donc curieux qu'au moment où les moyens de communication nous mettent le monde entier à 
portée de la main, les hommes de théâtre africains se barricadent derrière les accents sécurisants d'un passé souvent magnifié à tort. (30-31)

L'analyse de Kwahulé démontre que l'hybridité en tant que mélange des genres littéraires et artistiques fait partie du continent africain tout autant qu'elle en dépasse les frontières. L'évolution du théâtre africain est marquée par la rencontre de plusieurs formes hybrides forgées par l'influence des traditions et cultures orales, des rituels et discours politico-esthétiques sur la revalorisation de l'Afrique mais aussi des langues, des idéologies et techniques littéraires importées lors des colonisations. En effet, l'idéologie coloniale promulguée dans l'école primaire supérieure de Bingerville en Côte d'Ivoire et l'école normale supérieure de William Ponty au Sénégal dans les années 1930 favorise la mise en place de spectacles de théâtre "fête d'art indigène $»^{2}$. Les pièces de théâtre historiques et politiques anticoloniales dans les années 1950 et 1960 sont influencées par les théories de la négritude. Les esthétiques théâtrales négro-africaines (Didiga, Théâtre-rituel, Griotique, l'Ensemble Koteba) connaissent leur apogée dans les années 1970 et 1980. Tournées vers l'interdisciplinarité d'un art-total elles se placent dans la continuité de la lutte contre l'aliénation des cultures africaines engendrée par les colonisations. Citons également l'esthétique du Rocado Zulu Théâtre de Sony Labou Tansi, critique tragi-comique du néocolonialisme et des dictatures africaines qui participent également à la fabrication complexe du tissu hybride du théâtre africain. Qu'est-ce qui distingue alors l'hybridité des nouvelles écritures théâtrales africaines?

La rupture amorcée par un ensemble d'écrivains de la diaspora africaine dans le paysage théâtral depuis les années 1990 s'établit avec leur désir d'appréhender l'hybridité culturelle et esthétique sur le mode du mouvement et de la transformation permanente plutôt que sous la forme d'une essence africaine fixe. À l'instar des théoriciens des études postcoloniales dont la démarche consiste à déconstruire l'héritage du modèle de pensée coloniale de la représentation de l'Autre conçue dans l'obsession des binarités et de la fixité (Homi K. Bhabha), les nouveaux dramaturges africains se projettent dans le dépassement de toutes formes de discours d'autorité qui assignent l'africanité dans des champs cloisonnés. Cette position s'exprime par de multiples références aux notions d'entre-deux, d'entremultiple ou encore de traversée dans lesquelles ces dramaturges s'identifient pleinement porte le désir de se réinventer en dehors des catégories. Dans sa préface aux Nouvelles dramaturgies d'Afrique noire francophone, Caya Makhélé résume ainsi cette :

2 L'objectif de l'école Ponty était d'assurer la formation des futurs cadres africains de l'administration coloniale française. La section de théâtre encourageait les étudiants africains à se rendre dans des villages locaux et reporter les traditions dont ils devaient mettre en scène dans des spectacles et mémoires de fin d'année le caractère inopérant et dépassé par rapport aux valeurs portées par l'idéologie coloniale française. Les dramaturgies africaines issues de l'école Ponty sont dans la répétition des formes théâtrales classiques françaises. 
Écrivains entre diverses cultures, habitués à être définis par les autres, cherchant une définition de leur propre être. Désormais habités par leurs propres interrogations.

Écrivains en voyage à travers divers patrimoines et faisant exploser les frontières des préjugés sans pour autant jamais encore avoir eu une place qui ne soit toujours stipulée comme africaine.

Ecrivains porteurs de nouvelles émotions et formes pour les dire.

Écritures au cœur de différents exils. (10)

Les déplacements géographiques ou chemins d'exils en association avec les parcours professionnels sont en effet constitutifs de l'histoire de ces écrivains dont la majorité vit en France. Les itinéraires de ces dramaturges sont divers. José Pliya originaire du Bénin, est ancien directeur des Alliances Françaises (en Guinée Equatoriale, Cameroun, Dominique) puis directeur de l'Artchipel, Scène Nationale de Guadeloupe avant de s'installer aujourd'hui à Marseille où il dirige la compagnie de Diffusion, Production Internationale Caravelle ; Kossi Efoui a fui la dictature de l'ancien Président du Togo, Gnassingbé Eyadéma, et s'est installé à Amiens ; Koffi Kwahulé, qui après avoir commencé des études artistiques à l'Institut National des Arts à Abidjan, a continué des études théâtrales à Paris où il réside ; Caya Makhélé, née à Pointe-Noire au Congo, vit actuellement à Paris où il dirige la maison d'édition Acoria ; feu Marcel Zang a déménagé du Cameroun à un jeune âge avant de s'installer à Nantes; Dieudonné Niangouna, directeur artistique du festival international "Mantsina sur Scène" à Brazzaville, sa ville d'origine, vit aujourd'hui entre la France et le Congo. Le mouvement n'est toutefois pas seulement géographique et professionnel. Il s'inscrit dans le processus de l'écriture et de la construction identitaire en relation avec l'usage prédominant de notions relatives à l'étrangeté, la fragmentation et la terminologie du monstre chez ces auteurs.

\section{Identités monstres}

Je me sens comme une sorte de mutant : Je ne suis plus ni dans l'origine ni de l'autre côté ... . Il n'y a qu'un seul endroit où je me sens vraiment chez moi, en accord avec moi-même, c'est dans le rythme de ce que j'écris. C'est là que se trouve ma vraie identité. Je ne suis ni Africain, ni Camerounais, ni Français, je suis un produit de tout cela. (58-61)

Ces propos tenus par Marcel Zang au cours de la table ronde « Entre deux mondes, entre deux langues » s'inscrivent dans sa relation ambiguë au monde. Etranger à sa langue maternelle (le fang) qu'il ne parlait pas, étranger dans la langue (le français) de l'ancien colonisateur qu'il apprend sans avoir eu le choix, Zang exprime dans son théâtre la violence du rejet social et du sentiment d'étrangeté dans la société française. Cette société française est le reflet d'une Europe ethnocentriste qui vit dans la peur du mélange inévitable entre les peuples. Les personnages de ses 
pièces sont souvent des êtres mutants sur le plan psychologique à l'exception du personnage tératologique, "La Différence », décrit comme un "être étrange et distordu » (1) dans sa pièce cosmogonique Un Couple infernal (2010). Sa difformité est cependant présentée comme un avantage pour la perpétuation de l'hybridité dans un monde sclérosé et mourant. Le théâtre de Zang est peuplé de personnages noirs mutants en raison du fait qu'ils sont privés de leur langue maternelle et de la complexité de leurs racines culturelles en tant qu'immigrés à l'image d'Imago, le jeune sénégalais menacé d'expulsion dans L'Exilé (2002). Ce sont aussi des personnages souvent privés de leur liberté physique dans Bouge de là (2002) et La Danse du Pharaon (2004) mais qui luttent contre leur aliénation culturelle et mentale dans une société française qui refuse d'intégrer la mémoire et l'imaginaire des Africains dans son histoire postcoloniale.

Cette relation schizophrénique des anciennes puissances coloniales est définie par Kwahulé en tant que "syndrome Frankenstein", mentionné au début de notre étude. Kwahulé invite la référence au roman Frankenstein ; or The Modern Prometheus de Mary Wollstonecraft Shelley comme métaphore de l'hybridité forcée et orchestrée des peuples colonisés mais aussi de l'attitude méprisante néocoloniale. À l'instar du roman de Shelley où Frankenstein découvre avec horreur et mépris l'autonomie de sa créature qui s'est créé une nouvelle identité en devenant autodidacte, le "syndrome Frankenstein" désigne l'incapacité des anciennes puissances coloniales à sortir du regard exotique sur l'Autre et reconnaître la liberté et la puissance de re-création des écrivains africains comme faisant partie de l'héritage national. C'est un contexte similaire de révolte qui donne lieu à l'écriture du manifeste « Pour une littérature-monde en français » dans lequel plusieurs écrivains s'opposent à la distinction des institutions et des maisons d'édition entre les écrivains dits francophones et ceux dits français (sous-entendu "de souche"). L'écrivain Tahar Ben Jelloun résume parfaitement cette lutte dans le titre de son article «On ne parle pas le francophone» publié dans Le Monde diplomatique en mai 2017. Chez Kwahulé la créature (africaine) qui s'est partiellement construite à partir de l'influence coloniale/ Frankenstein a dépassé l'état de rébellion et de transgression de l'ordre dans sa capacité à reproduire son devenir dans les écritures de cette génération. Chalaye prolonge les références du devenir-monstre marqué historiquement par le sceau colonial mais plongé dans une dynamique de renouveau avec les nouvelles écritures théâtrales des dramaturges contemporains. Dans le numéro « Théâtres contemporains du Sud 1990-2006 » de Notre Librairie, Chalaye écrit :

tous enfants terribles des indépendances, ils ont érigé l'impertinence de Sony Labou Tansi en dynamique iconoclaste, celle de la culbute nécessaire : mettre sens dessus dessous les idées reçues, perdre le spectateur dans un univers où il ne retrouve plus les marques d'une identité repérable. Ils défendent un théâtre monstrueux et chimérique fait de marcottages et de collages, un théâtre qui soit le reflet de leur propre hybridation de colonisés, un « théâtre Frankenstein », un « théâtre alien ». (49) 
Menace provenant traditionnellement d'un ailleurs imaginaire ou d'origine surnaturelle dans les récits de science-fiction, l'alien est un mot anglophone dérivé du latin alienus signifiant "être étranger à quelqu'un ou à quelque chose". L'étrangeté de la créature du Dr. Frankenstein, assemblage de plusieurs parties de corps humains ramenées à la vie, représente au contraire le danger venu du dedans. La peur insoutenable de Frankenstein nourrit le maintien de la créature dans une altérité exotique radicale et l'absence de reconnaissance de son appartenance interne à la société. Ce n'est donc pas un hasard si un grand nombre de nouvelles dramaturgies de la diaspora africaine propose des personnages à l'identité instable recousue qui agissent aussi bien en tant qu'hybrides culturels que figures menaçantes de l'ordre établi. Prenons l'exemple de Attitude Clando (2007), pièce fondatrice qui a placé Dieudonné Niangouna au devant de la scène internationale lors de sa sélection dans la 71 eme édition du Festival d'Avignon. ${ }^{3}$ L'histoire met en scène un homme qui s'est évadé d'un hôpital psychiatrique où il a passé quinze ans. Sans attache et sans nom le protagoniste dissémine dans le texte ses souvenirs et certaines références à son passé (le pimentier dans la cour de son enfance, le départ forcé de sa terre natale, les mandats d'argent envoyés "au pays") qui suggèrent l'histoire d'un immigré clandestin. C'est un marginal dont l'identité en désordre apparaît comme une menace à l'équilibre réglé du système social. Il se confronte aux figures d'autorité et refuse de se soumettre aux règles en opposant la liberté de se réinventer en dehors des normes. Dans l'hôpital psychiatrique il déclare au médecin:

Je n'ai pas fini mon traitement d'intégration à la noix, docteur, je le sais. Mais je n'en ai pas besoin, je le sais aussi, c'est sûr que vous en avez mauvaise parce que je suis inachevé et que je ne sais pas lire les notices. Mais je préfère ma posologie perso. Alors vous savez donc que je n'ai nul besoin de vous parce que vous avez besoin de moi. (79)

Dans le discours du clandestin se profile le constat lucide de sa condition monstrueuse. Le thème de l'inachèvement indiqué dans la citation et repris continuellement tout au long de la pièce comme dans un grand nombre de nouvelles dramaturgies africaines décrit l'écart normatif utilisé comme arme de coercition et d'exclusion sociale. Les conditions de détention du clandestin dans l'hôpital psychiatrique évoquent à la fois la mise à distance et l'exhibition du corps de l'étranger (l'alien) devenu objet de curiosité et d'analyse scientifique.

Je vous interdis de rentrer dans ma vie brisée. La taille de mon sexe ne regarde que ma femme. Vous m'avez mis nu plusieurs fois dans votre hôpital pour savoir comment j'évoluais. J'étais nu, la boule à zéro, et vous me rinciez tendrement avec un tuyau d'eau chaude pour tuer les microbes. Et maintenant vous voulez que je replonge ? Pourquoi faire ? (78)

Mensurations et observations de l'anatomie sous toutes les coutures, traitement dans des conditions proche de l'animalité, ces descriptions des pratiques

\footnotetext{
${ }^{3}$ Niangouna devient alors le premier artiste africain associé au Festival d'Avignon.
} 
intrusives par le corps médical ne sont pas sans rappeler les discours scientifiques sur la hiérarchie des races qui ont justifié l'organisation du système esclavagiste et colonial à l'aide des expositions et spectacles ethnologiques dits "zoos humains". En d'autres termes, le monstre dans ce contexte est un être "racialisé" synonyme de créature sauvage ou indigène qu'il faut domestiquer par son intégration. La mise en spectacle ou monstration du monstre sauvage dans les zoos humains justifie en elle-même l'idéologie de sa domination comme l'explique entre autre Pascal Blanchard dans l'ouvrage collectif Zoos humains. De la Vénus Hottentote aux reality shows: " la mise en scène se suffit à elle-même et fait passer, bien plus efficacement parce que souterrainement, le principe raciste qui la fonde » (6). Le « clando » de Niangouna refuse de participer à la spectacularisation de son corps classé en tant que figure antithétique de l'altérité et pose à la place les conditions du développement créatif de soi par l'inachèvement et la fragmentation identitaire. Il se réapproprie l'image du monstre pour la renverser en une figure contemporaine de résistance sociale qui rejette toute forme d'identification à l'origine du processus social d'aliénation des individus.

Je paie tout au noir, j'ai pas de carte bancaire, pas de chèque à laisser à la machine, pas de numéro de Sécu sociale, j'ai pas de carte d'identité, pas d'assurance maladie, et pas de psychologue à juger de la raison de mes neurones. J'ai pas de téléphone portable, pas de fixe, ni de fax, pas d'adresse électronique, je ne vais jamais à la poste, jamais au supermarché, j'évite toujours les caméras et prends jamais de photos, je mets des gants pour ne pas laisser mes empreintes, je ne vais jamais dans les milieux où la logique m'attend, interdiction formelle de toucher à un ordinateur. J'ai même réussi à ne pas avoir une signature et à oublier mon nom. (71-72)

Ou encore lorsque le « clando » déclare: « Suis hors pair, hors marge ni dans la page, hors périmètre, suis pas à la surface, inachevé, fort à mon aise » (85).

Le titre de la pièce de Niangouna confirme un mode de vie clandestin en tant que posture au monde. Attitude Clando porte les aspirations d'une génération non pas à sombrer dans l'anonymat mais à porter une nouvelle visibilité libérée des carcans et des étiquettes sur l'africanité. La structure de la pièce se fait le relais de l'espace mental chaotique du personnage principal. Il n'y a pas d'indication scénique ni liste des personnages. Bien que divisée en trois parties la pièce se lit comme une logorrhée discontinue de réflexions et souvenirs épars qui est prononcée du point de vue du « clando » sans que l'on sache véritablement s'il y a un destinataire. Le texte s'achève sur une phrase exclamative au sujet d'un coup de feu sans expliquer ce qui arrive au personnage. Cette dernière phrase ou action du « clando » reste donc indéchiffrable à la mesure de l'impossible identification du protagoniste dans la pièce. Niangouna engage une discussion ouverte sur le sens de la clandestinité par-delà le thème de l'immigration. Le mode de vie clandestin est assumé au sein de la violence exercée par une société, culture ou groupe qui impose ses règles dans ce que le clando appelle « le programme d'épuration des types pas réglos » (89). Les raisons de l'internement du « clando » ne sont jamais expliquées dans la pièce qui se focalise sur la violence institutionnelle des sociétés 
contemporaines. Le personnage monstre dans ces nouvelles dramaturgies est constamment en lutte contre un système. Conforme à aucun modèle il noue un rapport au monde par effraction afin de se réapproprier le discours sur son image identitaire.

\section{Du chaos au monstre}

Espace du multiple et l'irrégularité, le chaos est défini conventionnellement soit comme le vide béant ou l'état de confusion originelle des éléments précédant l'organisation du monde. Le chaos est souvent interprété en tant que désordre qui appartient à l'ordre de l'irrationnel et de l'anormal. La suspicion, la peur voire le dégout sont régulièrement associés à la condition chaotique. Dans Images et symboles : Essais sur le symbolisme magico-religieux, Mircéa Eliade explique la naissance des rhétoriques du centre dans les sociétés traditionnelles et modernes qui se sont toujours méfiées du chaos perçu comme une menace de l'inconnu. « Nombre de textes assimilent les adversaires en train d'attaquer le territoire national, aux larves, aux démons, ou aux puissances du chaos » (17) souligne Eliade. Les expressions théâtrales négro-africaines telles que le Kotéba, le Didiga, le théâtre-rituel du Ki-Yi Mbock, et le Bin Kadi So s'inscrivent dans une transformation du chaos africain en un retour au centre qui concentre la quête d'un espace social, politique et culturel en harmonie avec l'individu et le collectif. C'est dans ce sens que Liking emprunte le symbole de l'étoile à cinq branches du Ki Yi chez les bassa du Cameroun pour représenter la perfection de l'équilibre de vie. L'état chaotique africain inspire la peur en même temps qu'il fascine et c'est dans le théâtre politique qu'il prend la forme d'une critique avec les littératures contestataires contre les empires coloniaux entre les années 1950 et 1970 puis d'une autocritique post-indépendante. L'écriture du chaos devient une arme pour dénoncer les monstres de la société et des structures politiques dominantes. L'écrivain iconoclaste Sony Labou Tansi a particulièrement mis en lumière cette nature monstrueuse dans la représentation du " chaos du réel » (Tchak 112) dans ses pièces de théâtre et romans qui dénoncent la violence politique et sociale des gouvernements militaires et des partis uniques qui ont succédé aux politiques coloniales. Le chaos chez Labou Tansi est représenté par la récurrence de personnages monstrueux du point de vue éthique (dictateurs, bourreaux, militaires sanguinaires, politiciens avides de pouvoir) responsables de la déréliction des sociétés où " les lois n'ont plus de conscience » (La Parenthèse de sang 21). Ce monde chaotique est relayé par la fabrication du désordre au sein du style littéraire de Labou Tansi qui bouscule volontairement la langue française en détournant ses codes classiques. ${ }^{4}$ L'enjeu est en effet de faire résonner les mots autrement et de saisir l'imaginaire des peuples face à la progression d'un "cosmicide" engendrés par des politiques du mensonge, de la violence, du racisme et de l'exploitation des individus. L'écriture laboutansienne se place à la fois dans une interprétation

${ }^{4}$ L'écriture de Sony Labou Tansi est marquée par l'usage abondant d'images métaphoriques, de néologismes et de tournures ironiques qui accentuent le désordre et le grotesque du pouvoir politique autoritaire. 
classique et innovante du chaos qui représente d'une part l'absence de règles et de moralité à l'image des personnages de monstres sanguinaires et d'autre part l'espace multiple monstrueux de la subversion syntaxique et structurelle.

Héritiers du théâtre désenchanté de Sony Labou Tansi, les dramaturges de la diaspora africaine à partir des années 1990 formulent de nouvelles scénographies du chaos monstrueux non plus sur le plan éthique mais en tant que processus de renouvellement identitaire. Pris dans de nouvelles préoccupations sur la nécessité de reformuler la problématique de l'identité (comme pour mieux l'évacuer) à partir de la réalité du multiple, de la fragmentation et du « déracinement» (Traoré 149), ils introduisent la monstruosité chaotique en tant qu'espace complexe de liberté et de réinvention. Les écritures théâtrales de la diaspora africaine reflètent le monstre hybride dans la visibilité de personnages hors normes en lutte contre leur invisibilité sociale à l'aide de constructions énonciatives et d'esthétiques chaotiques. La mutation des dramaturgies africaines à partir des années 1990 désigne principalement une rupture esthétique subversive dans les écritures théâtrales du chaos africain. Dans Traversée de la subversion: Les dramaturgies d'expression française, Dany Toubiana explique que les théâtres en français sont animés par le désir commun de subvertir les modèles établis depuis la lutte des indépendances politiques et sociales. Cette subversion vise « au renversement d'un ordre établi par rapport au cadre théâtral, aux modèles, aux personnages et à la langue » (Toubiana 28). La mutation des nouvelles dramaturgies africaines remet en cause les conventions du genre théâtral africain et fait émerger l'étrangeté et le monstrueux dans une récupération transformative des éléments du chaos. À l'instar des pièces de Sony Labou Tansi, ces nouvelles dramaturgies proposent de repenser la représentation du chaos du réel mais dans un mouvement permanent de l'expérience diasporique africaine. Le Carrefour (1989), pièce inaugurale qui amorce cette rupture de la nouvelle génération est interprétée comme un « ovni » (Chalaye, 8) face à une critique qui ne retrouve pas dans l'œuvre de Kossi Efoui les codes traditionnels de lecture du théâtre africain. Dans cette pièce en rhizome, Efoui présente un ensemble de récits croisés chez des personnages errants et meurtris par les violences d'un gouvernement autoritaire et situés dans un carrefour de la ville. Ce carrefour de dialogues et de rencontres dans un espace-temps cyclique est une métaphore de la résistance de la mémoire créative du personnage principal (Le Poète) qui réinvente les souvenirs de l'ensemble de ces récits et personnages dans la prison où il croupit depuis vingt ans. Dans cette forme narrative polyphonique, dont il faudra expliquer la récurrence dans les dramaturgies contemporaines africaines, ce n'est pas seulement le chaos mental du protagoniste qui est mis en relief mais l'impossibilité de mettre en scène des personnages monstres autrement que dans une esthétique du chaos.

\section{Nouvelles esthétiques du chaos monstrueux}

Le retour de la question identitaire (à savoir la définition de l'africanité) dans la nouvelle vague de dramaturges africains s'entend dans le paradoxe de son dépassement. Le chaos dans l'écriture devient donc le cadre idéal pour retranscrire la cohérence des lignes de fuites de l'identité. C'est que l'identité même du chaos 
en tant que dynamique des systèmes non-linéaires est en réalité plus complexe et organisée qu'elle ne paraît. L'historien des sciences James Gleick, dans son bestseller Chaos: Making a New Science publié en 1987, explique en effet que l'évolution des théories du chaos dans les sciences (la météorologie, la chimie, la physique, la médecine, entre autres) et les sciences sociales telles que la sociologie, la philosophie ou l'économie met en lumière des lois de stabilité qui régissent le désordre de l'espace chaotique. En d'autres termes, le chaos n'est pas l'absence d'ordre mais la configuration d'un état normal instable du monde dont il faut apprendre à saisir le sens et la régularité affirme Katherine Hayles dans Chaos and Order: Complex Dynamics in Literature and Science. Gleick rend compte de la fin des siècles hégémoniques du système newtonien qui plaçait le déterminisme linéaire au centre de la compréhension de l'univers et définissait le multiple de l'espace chaotique contre nature. Cette nouvelle compréhension identitaire du chaos trouve un écho dans le théâtre de la diaspora africaine depuis les années 1990. En effet, l'articulation d'une pensée sur les conditions régulières de l'identité plurielle dans un mouvement perpétuel de transformation de la mémoire individuelle et collective engage le passage à de nouvelles scénographies du chaos chez les nouveaux dramaturges. Les structures du chaos telles que la fragmentation, la répétition et la liminalité deviennent des outils de narration pour comprendre et analyser les dramaturgies contemporaines africaines. Si le chaos dans l'écriture devient plus lisible, il n'en demeure pas moins monstrueux de par l'objectif de se libérer des normes établies. Le monstre ne se lit plus seulement dans des personnages rebelles tels que le « clando » mais dans la forme chaotique même du texte. Lope de Vega évoquant le « monstro cómico » (le monstre comique) dans $E l$ Arte nuevo de hacer comedias (1609) et Corneille 1'“étrange monstre" pour qualifier l'Illusion comique (1636) s'engageaient déjà dans la défense d'une esthétique théâtrale monstrueuse lorsqu'ils décrivaient le style nouveau de la tragicomédie. À l'instar des deux dramaturges du XVIIème siècle dont les idées s'inscrivaient à contre-courant des théories fixistes traditionnelles des genres (la Poétique d'Aristote), les dramaturges africains à partir des années 1990 opposent des esthétiques du chaos monstrueux libérées du déterminisme linéaire d'une pensée essentialiste sur l'africanité. Toutefois, l'écriture monstrueuse des dramaturges africains ne se pose pas simplement comme un plaidoyer pour le mélange des genres littéraires (comme chez de Vega et Corneille) mais comme la reconnaissance de la fragmentation identitaire dans le champ du multiple. Comment la monstruosité chaotique de ces écritures théâtrales est-elle organisée ?

Les nouvelles esthétiques théâtrales africaines du chaos sont marquées par la récurrence de certaines structures telles que l'instabilité des repères spatiotemporels, la superposition des espaces et des récits et la fragmentation des voix narratives. Prenons l'exemple de l'un des écrivains les plus prolifiques de cette génération, Koffi Kwahulé, auquel la critique a consacré un grand nombre d'essais. Inspirée par l'univers musical des professionnels noirs américains du jazz tels que John Coltrane, Thelonious Monk, Dizzy Gillespie, et Billie Holiday, l'écriture de Kwahulé est rythmée par des jeux de syncopes et d'inflexions qui donnent l'impression d'une forme improvisée. La musique jazz inspire tout autant ses pièces 
de jeunesse, $1+1=1$ (1981), Le Grand Serpent (1982) que celles qui ont directement suivi, telles que Cette vieille magie noire (1993), Bintou (1996) ou Il nous faut l'Amérique (1997). L'éclatement de la forme dramatique toutefois s'est accentué avec l'écriture de Jaz (1998) puis dans les œuvres postérieures. Structurée par un long monologue dont la construction syntaxique est disjointe, Jaz raconte l'histoire traumatique d'une femme violée. L'absence d'indication des personnages dans la pièce est remplacée par les indications scéniques préliminaires suivantes:

Une femme.

Le crâne rasé peut-être.

Nue peut-être.

Un révolver.

Des balles

Une ardoise.

Un jazz (un seul instrument)

Qui de temps à autre,

Trou/est troué

enlacé/est enlacé

par la voix de la femme. (57)

La construction syntaxique des indications scéniques de Kwahulé met en relief un rythme saccadé et décousu des phrases et de la ponctuation qui plonge le lecteur dans la fragmentation du récit. L'incertitude sur les caractéristiques physiques du personnage principal (femme peut-être au crâne rasé ou peut-être nue) se poursuit dans la tension narrative de la pièce dans laquelle une femme répète inlassablement « Je ne suis pas ici pour vous parler de moi mais de Jaz ». L'histoire nous apprend cependant que cette femme qui évoque avec difficulté les viols répétés qu'elle a subis n'est autre que Jaz. Les procédés de distanciation opérés dans le discours de Jaz la placent à la fois comme étant présente et absente de la narration. La personnification de l'instrument de musique jazz présent sur scène ne joue pas la fonction de simple accompagnant mais se mêle à la parole traumatique du personnage. Il devient le prolongement du corps de Jaz. La parole musicale intervient donc pour "trouer le discours" (95) selon l'expression de Jean-Godefroy Bidima et maintenir ainsi l'équilibre instable de la narration chaotique monstrueuse. L'exposition de la première scène du viol de Jaz dans les toilettes publiques met en scène un ensemble de constructions syntaxiques en rupture qui traduisent la violence de l'événement dans un rythme musical discontinu et accéléré.

Elle a glissé une pièce

dans la fente

la porte s'est ouverte

et elle s'est sentie brutalement poussée dans la cabine

Par l'homme

Il a immédiatement renfermé la porte après eux.

Non.

Elle n'a pas crié non plus. 
Parce qu'elle ne comprenait pas ce qui se passait

L'esprit patine dans ces moments-là

Déshabillez-vous il a dit à Jaz.

La voix se voulait douce et

le ton un rien obséquieux.

Pour peu il aurait ajouté s'il vous plait.

Etrangement.

Un couteau.

Un couteau de cuisine

Au début oui.

Evidemment

Etre vouvoyée dans une sanisette

un couteau entre les cuisses

ne peut être rassurant pour personne

Jaz s'est déshabillée

Votre culotte

Parce que Jaz avait peur de la peur de l'homme.

Très. (72)

Dans cet extrait, l'alternance des focalisations internes et externes produit un effet de rétrécissement de la scène qui semble s'improviser sous les yeux du lecteur. L'usage de phrases courtes sur le mode quasi impératif et de phrases nominales donne une accélération au récit. La narration est amplifiée par la structure polyphonique des voix (celle de Jaz, du violeur, et de Jaz en tant que narratrice) qui se superposent dans le monologue. Le placement aléatoire, en apparence, des adverbes (étrangement, évidemment, très) ponctue le discours en opérant une stratégie de subversion grammaticale du monologue. Ces ruptures dans la narration construisent une oralité de la mémoire en action. Jaz opère une construction de la mise en forme de l'identité chaotique monstrueuse. L'ellipse du second $\mathrm{z}$ dans le nom du personnage évoque une distorsion de la musique jazz à l'intérieur du texte. Dans l'ouvrage Frères de son, Koffi Kwahulé et le jazz: entretiens Kwahulé du musicologue Gilles Mouëllic, Kwahulé explique cette perte du z:

L'absence du z signifie l'amputation irrémédiable que l'on ressent après l'expérience traumatique du viol. Violer, c'est amputer profondément et durablement. C'est aussi le vertige de se retrouver au bord du précipice que laisse à jamais en soi l'éboulement soudain de son identité. Enfin, et plus simplement, l'absence de z montre, au sens photographique du terme, le manque, l'absence dans laquelle s'enracine le jazz. Car malgré cette différence entre mon écriture et le jazz, Jaz nous plonge bel et bien au cœur du jazz. (28)

Le monologue à plusieurs voix ou "polylogue" pour reprendre l'expression de Jean Pierre Sarrazac peut se comprendre en tant que jaillissement imprévisible de la mémoire oubliée à partir des résonnances chaotiques d'histoires collectives et individuelles. La parole jazzistique de Jaz peut donc s'interpréter dans la 
représentation allégorique du combat contre la désappropriation identitaire, culturelle et économique des Africains pendant l'esclavage et la colonisation et des Noirs Américains pris dans l'étau des violences raciales systémiques par exemple.

Philippe Carles et Jean-Louis Comolli rappellent que la musique free jazz s'est construite aux Etats-Unis dans les années 1960 en réaction contre une pratique du jazz jouée par des Noirs mais colonisée par les Blancs sur le plan économique et culturel.

Le free jazz apparaît à la fois comme résistance à cette expropriation, réaction de rejet des valeurs (musicale et extra-musicales) de l'idéologie dominante (aux U.S.A., capitaliste et blanche), tentative de libération culturelle en écho des luttes des Noirs américains pour leur libération politique et économique, entreprise de reconquête et de construction d'une culture spécifique afro-américaine. (50)

Le viol de Jaz peut illustrer ce thème de l'expropriation culturelle et identitaire souligné par Carles et Comolli et l'écriture chaotique monstrueuse de Kwahulé réussit à maintenir une tension collective à l'échelle d'un individu, personnage diasporique dont l'identité est reconstituée dans un ensemble de fragments. Jaz habite dans une cité insalubre délaissée des pouvoirs publics parmi une population assommée par la misère économique. Son personnage incarne le seul élément de beauté et de résistance sociale. L'origine de Jaz n'est pas dans une indication géographique (qui n'est jamais donnée dans la pièce) mais dans la structure éclatée d'une écriture en mouvement et imprévisible. Jaz concrétise le projet du meurtre $\mathrm{du}$ violeur dans la scène finale et poursuit le processus de réappropriation de son identité. La monstruosité n'est donc pas dans la revanche de Jaz mais dans la mise en forme chaotique du personnage qui laisse apparaître un calligramme dans le texte. Judith Miller qui interprète la forme de ce calligramme comme celle d'une « femme-oiseau» (92) confirme l'idée du monstre chaotique hybride dans l'écriture comme ouverture du champ des possibles et de la réinvention de soi. En effet le calligramme de Jaz, d'après Miller, exprime la liberté de cette femme « qui ne sera jamais unifiée mais qui trouvera une certaine plénitude dans la polyphonie chorale si bien orchestrée par Kwahulé » (93).

Esthétique théâtrale de l'identité plurielle, la monstruosité chaotique se comprend d'abord comme mode d'existence chez cette nouvelle génération d'écrivains qui assument les conditions de l'être hybride. C'est dans cette posture que Léonora Miano explique les "identités frontalières" inspirées des théories postcoloniales:

Être un Africain, de nos jours, c'est être un hybride culturel. C'est habiter la frontière. Le reconnaître, c'est être honnête envers soi-même, regarder en face ses propres réalités, et être capable de les infléchir. Il ne s'agit pas de chercher à valoriser l'une ou l'autre des composantes de cette identité, mais de se dire qu'on a le privilège rare de pouvoir choisir le meilleur de chaque culture. (30) 
La mutation du théâtre de la diaspora africaine se poursuit dans la reconstruction d'espaces ouverts de l'africanité en mouvement. Le monstre chaotique et protéiforme des écritures théâtrales contemporaines africaines remet en cause les discours essentialistes qui reposent sur le principe fondateur d'unité. Définit en dehors des normes c'est un monstre qui affirme cependant sa place à l'intérieur du monde dans une résistance contre toute tentative d'invisibilité de sa parole. Les nouveaux dramaturges sont animés par le désir de penser librement la création africaine au détour des influences culturelles transnationales. L'enjeu est bien de se réapproprier un espace de l'imaginaire et penser le renouvellement de la création africaine à partir de nouvelles sonorités dans le langage.

\section{Bibliographie}

Ben Jelloun, Tahar. « On ne parle pas le francophone. » Le Monde diplomatique. Mai 2017.

Blancel, Nicolas, Pascal Blanchard, Gilles Boetsch, Eric Deroo, et Sandrine Lemaire. Zoos humains. De la Vénus Hottentote aux reality shows. Paris: La Découverte, 2002.

Bidima, Jean Godefroy. La Philosophie négro-africaine. Paris: Presses Universitaires de France, 1995.

Carles, Philippe et Jean-Louis Comolli. Free Jazz Black Power. Paris: Gallimard, 2000.

Chalaye, Sylvie. Introduction. Afrique noire et dramaturgies contemporaines: le syndrome Frankenstein. Édité par Sylvie Chalaye. Paris: Théâtrales, 2004. 
---. « Dramaturgies de la culbute et du détour. » Théâtres contemporains $d u$ Sud 1990-2006, vol. 162, 2006, pp. 47-53.

---. Introduction. Le Théâtre de Kossi Efoui: une poétique du marronnage. Éd. Sylvie Chalaye. Paris: L'Harmattan, 2011, pp. 8-9.

« Dramaturgies africaines d'aujourd'hui. » Les petits matins du TOMA, La Chapelle du Verbe incarné, Avignon, le 23 juillet 2001. Table ronde.

Efoui, Kossi. Préface. L'Entre-deux rêves de Pitagaba conté sur les trottoirs de la radio. Paris: Acoria, 2000.

Eliade, Mircéa. Images et symboles: Essais sur le symbolisme magico-religieux. Paris: Gallimard, 1952.

Gleick, James. Chaos: Making a New Science. New York City: Viking Press, 1987.

Hayles, Katherine N. Chaos and Order: Complex Dynamics in Literature and Science. Chicago: University of Chicago Press, 1991.

Jefferson, Louise M. Introduction. Théâtre noir francophone. Euvres et critiques XXVI, 2001, pp. 5-14.

Kwahulé, Koffi. "Quand l'africanisme dérive vers l'intégrisme culturel: le cas de la Côte d'Ivoire". Théâtres d'Afrique noire. Alternatives théâtrales, vol. 48, 1995, pp. 30-31.

---. Jaz. Paris: Théâtrales, 1998.

Kwahulé, Koffi et Gilles Mouëllic. Frères de son. Koffi Kwahulé et le jazz: entretiens. Montreuil-sous-Bois: Théâtrales, 2007.

Makhélé, Caya. Introduction. Théâtres d'Afrique noire. Alternatives théâtrales, vol. 48, 1995.

---. Préface. « L'art du risque. » Nouvelles dramaturgies d'Afrique noire francophone. Éd. Sylvie Chalaye. Rennes: Presses Universitaires de Rennes, 2004, pp. 9-14.

Miano, Léonora. Habiter la frontière. Paris: L'Arche, 2012.

Miller, Judith. « La choralité du monologue chez Koltès et Kwahulé. » Fratries Kwahulé: Scène contemporaine chœur à corps. Paris: L'Harmattan, 2009, pp. 86-93.

Niangouna, Dieudonné. Attitude Clando, Paris: Cultures France, 2007.

Toubiana, Dany. Traversée de la subversion : Les dramaturgies d'expression française. Paris: L'Harmattan, 2010.

Tchak, Sami. Paroles inédites. Dir. Bernard Magnier. Montreuil-sous-bois: Editions Théâtrales, 2005.

Traoré, K. Dominique. Dramaturgies d'Afrique noire francophone, dramaturgies des identités en devenir. Paris: Le Manuscrit, 2008.

Zang, Marcel. Un couple infernal. AgoraVox 11 septembre 2010. www.agoravox.fr/tribune-libre/article/un-couple-infernal-80989. Accédé le 12 décembre 2017. 